\section{Disparidades nas filas para transplantes de órgãos nos estados brasileiros}

\author{
Geographic disparities in organ transplantation \\ in Brazil
}

\author{
${ }^{1}$ Instituto de Pesquisa \\ Econômica Aplicada, Rio de \\ Janeiro, Brasil. \\ Correspondência \\ A. Marinho \\ Instituto de Pesquisa \\ Econômica Aplicada. \\ Av. Presidente Antonio Carlos \\ 51, 10 o andar, Rio de Janeiro, \\ RJ 20020-010, Brasil. \\ amarinho@ipea.gov.br
}

\begin{abstract}
This study focuses on organ transplantation in Brazilian States in the years 2004, 2005, and 2006. Average waiting times were estimated by queueing theory models. We found that average waiting time for transplantation decreased for cornea and pancreas, increased for liver, heart, and kidney/ pancreas, and varied erratically for kidney and lung. We also detected broad disparities in waiting times between States. Mean waiting time was shortest in the States of South, Southeast, and Midwest Brazil. These same States also showed the highest transplantation rates in the country.
\end{abstract}

Organ Transplantation; Healthcare Disparities; Health Policy
Alexandre Marinho 1 Simone de Souza Cardoso 1 Vivian Vicente de Almeida 1

\section{Introdução}

O fenômeno de disparidade geográfica em transplantes de órgãos é amplamente relatado na literatura 1 . Existem relatos do problema em países desenvolvidos como Austrália 2, Espanha 3, Estados Unidos 4,5, França 6 e Reino Unido 7.

No Brasil, as eventuais conseqüências desse fenômeno podem ser agravadas pelas dimensões continentais do país. Entretanto, não existem indicadores oficiais gerais do tempo de espera nas filas e de outras variáveis relevantes relacionadas aos transplantes de órgãos no Brasil. O presente trabalho atualiza e estende o trabalho de Marinho 8, que avalia aspectos econômicos das filas, e apresenta um conjunto de estimativas relacionadas com o tempo de espera para alguns órgãos e tecidos (coração, córnea, fígado, pulmão, rim, pâncreas e transplante simultâneo de rim e pâncreas) no Sistema Nacional de Transplantes (SNT). Os resultados obtidos estimam tempos de espera que, mesmo em um modelo otimista, quase sempre ultrapassam um ano e que, em um modelo menos otimista, poderia atingir, por exemplo, quase nove anos para fígado e mais de 11 anos para rim. Mais ainda, esse tempo de espera é superior a alguns indicadores internacionais (relacionados na Tabela 1). Esses dados se tornam ainda mais relevantes quando observamos a baixa porcentagem existente entre a quantidade de transplantes realizados e a demanda por transplantes, conforme a Tabela 2, também 
Tabela 1

Tempo de espera (em anos) para transplantes em países selecionados.

\begin{tabular}{|c|c|c|c|c|c|}
\hline \multirow[t]{2}{*}{ Órgãos } & \multicolumn{2}{|c|}{ Estados Unidos * } & \multicolumn{2}{|c|}{ Reino Unido ** } & \multirow[t]{2}{*}{ SUS *** } \\
\hline & Brancos & Negros & Adultos & Crianças & \\
\hline Coração & 0,41 & 0,36 & 0,28 & 0,39 & 0,83 \\
\hline Fígado & 1,02 & 0,45 & 0,26 & 0,21 & 4,41 \\
\hline Pulmão & 1,59 & 2,07 & 1,11 & ND & 1,77 \\
\hline Rim & 3,59 & 5,02 & 2,30 & 0,45 & 5,53 \\
\hline Rim/Pâncreas & 6,67 & 1,47 & 0,72 & ND & 1,32 \\
\hline Pâncreas & 1,30 & 3,28 & ND & ND & 2,63 \\
\hline
\end{tabular}

ND: não disponível; SUS: Sistema Único de Saúde.

* Medianas dos pacientes alistados em 2003 e 2004, de acordo com a Organ Procurement and Transplantation Network (OPTN). http://optn.transplant.hrsa.gov/latestData/rptStrat.asp (acessado em 03/Jun/2009);

** Mediana nos períodos 2000-2003 (rim); 2000-2004 (coração e pulmão); 2002-2005 (fígado); e 2002-2005 (pâncreas e rim),

de acordo com a NHS Blood and Transplant 24. Menos de 100 observações para coração em crianças;

*** Médias estimadas para 2003 de acordo com Marinho 8.

apresentada a seguir e analisada com mais detalhe posteriormente no texto. São muitas pessoas a esperar muito tempo, com desiguais condições de acesso aos transplantes de órgãos no Brasil.

Mais precisamente, o presente texto, que é uma extensão aos estados brasileiros do referido trabalho de Marinho 8, avalia a disparidade de acesso aos transplantes de órgãos entre as Unidades da Federação (estados e Distrito Federal), no que se refere aos diferentes tempos de espera para os transplantes. Descentralizamos o cálculo do tempo de espera nas filas para transplantes de órgãos (rim, córnea, coração, fígado, pulmão, pâncreas, rim/pâncreas) para os estados (com exceção do Acre, sem dados de produção no período) e para o Distrito Federal (DF), para os anos de 2004, 2005 e 2006.

\section{Breve descrição do SNT}

De acordo com a página do Ministério da Saúde na Internet (http://portal.saude.gov.br/por tal/saude/area.cfm?id_area=1004), o SNT, criado em 1997, é o responsável pela administração dos transplantes financiados pelo Sistema Único de Saúde (SUS) no Brasil. O SNT dispõe de 25 Centrais de Notificação, Captação e Doação de Órgãos (CNCDO) nos estados e no Distrito Federal, e de uma Central Nacional de Notificação Captação e Doação de Órgãos (CNNCDO), localizada em Brasília. Além disso, dispõe de 548 estabelecimentos autorizados a realizar transplantes, envolvendo 1.376 equipes médicas.

O SNT é um dos maiores sistemas públicos de transplantes do mundo, e nenhum sistema de preços é relevante para selecionar os candidatos à recepção de órgãos no Brasil. O Brasil é o segundo país com maior número de transplantes, atrás apenas dos Estados Unidos, que fizeram 27.961 no ano de 2008, com 101.944 pessoas aguardando nas filas em 30 de abril de 2009 (United Network for Organ Sharing. http://www.unos. org/Data/default.asp?displayType=usData). Vale ressaltar que, nos Estados Unidos, os pacientes pagam pelos transplantes diretamente, ou por meio de planos de saúde, com exceção dos muito pobres, que recebem financiamento dos programas governamentais assistenciais (Medicare e Medicaid), conforme a Joint Commission on Accreditation of Healthcare Organizations (JCAHO) ${ }^{9}$. De acordo com a página do SNT na Internet (http://dtr2001.saude.gov.br/transplan tes/index_gestor.htm), no ano de 2008, foram realizados no país 19.125 transplantes de diversos órgãos e de tecidos. Foram implantados 12.825 córneas, 3.154 rins, 1.110 fígados, 43 pâncreas, 205 corações, 53 pulmões. Foram realizados 127 transplantes simultâneos de rim/pâncreas e 26 transplantes simultâneos de fígado/rim. Ocorreram, ainda, 1.582 transplantes de medula óssea. Outros órgãos e tecidos, como válvulas cardíacas, ossos, veias, tendões, pele e intestino, também podem ser transplantados. A fila de espera para transplantes totalizava 68.906 pessoas no ano de 2006. O gasto com transplantes, incluindo medicamentos, no ano de 2005, foi de $\mathrm{R} \$ 521,8 \mathrm{mi}$ lhões, ou seja, $29,11 \%$ a mais do que os $\mathrm{R} \$ 404,41$ milhões gastos em 2004.

No Brasil, o transplante de órgãos, por doação ao Estado, somente pode ser feito após a morte encefálica do doador, que pode ser natural ou 
Tabela 2

Quantidade de pessoas na fila de espera para transplantes, quantidade de transplantes para órgãos selecionados, e razão de atendimento, no ano de 2006.

\begin{tabular}{|c|c|c|c|c|c|c|c|c|c|c|c|c|}
\hline \multirow{2}{*}{$\begin{array}{l}\text { Unidade da } \\
\text { Federação }\end{array}$} & \multicolumn{3}{|c|}{ Rim } & \multicolumn{3}{|c|}{ Córnea } & \multicolumn{3}{|c|}{ Coração } & \multicolumn{3}{|c|}{ Fígado } \\
\hline & $\mathbf{L}$ & Tx & $\begin{array}{c}\mathrm{Tx} / \\
(\mathrm{Tx}+\mathrm{L}) \\
(\%)\end{array}$ & $\mathbf{L}$ & Tx & $\begin{array}{c}\mathrm{Tx} / \\
(\mathrm{Tx}+\mathrm{L}) \\
(\%)\end{array}$ & $\mathbf{L}$ & $\mathrm{Tx}$ & $\begin{array}{c}T x / \\
(T x+L) \\
(\%)\end{array}$ & $\mathrm{L}$ & Tx & $\begin{array}{c}\mathrm{Tx} / \\
(\mathrm{Tx}+\mathrm{L}) \\
(\%)\end{array}$ \\
\hline Alagoas & 704 & 25 & 3,43 & 269 & 18 & 6,27 & 2 & 0 & 0,00 & 0 & 0 & SA \\
\hline Amazonas & 386 & 23 & 5,62 & 470 & 68 & 12,64 & 0 & 0 & SA & 0 & 0 & SA \\
\hline Bahia & 1.972 & 54 & 2,67 & 603 & 98 & 13,98 & 0 & 0 & SA & 234 & 14 & 5,65 \\
\hline Ceará & 398 & 109 & 21,50 & 1.407 & 210 & 12,99 & 8 & 12 & 60,00 & 159 & 46 & 22,44 \\
\hline Distrito Federal & 526 & 34 & 6,07 & 1.209 & 276 & 18,59 & 0 & 0 & SA & 0 & 0 & SA \\
\hline Espírito Santo & 844 & 55 & 6,12 & 329 & 91 & 21,67 & 3 & 0 & 0,00 & 10 & 10 & 50,00 \\
\hline Goiás & 507 & 72 & 12,44 & 1.858 & 795 & 29,97 & 13 & 2 & 13,33 & 0 & 0 & SA \\
\hline Maranhão & 390 & 30 & 7,14 & 359 & 58 & 13,91 & 0 & 0 & SA & 0 & 0 & SA \\
\hline Mato Grosso & 848 & 1 & 0,12 & 395 & 39 & 8,99 & 2 & 0 & 0,00 & 0 & 0 & SA \\
\hline $\begin{array}{l}\text { Mato Grosso } \\
\text { do Sul }\end{array}$ & 272 & 49 & 15,26 & 147 & 90 & 37,97 & 16 & 0 & 0,00 & 0 & 0 & SA \\
\hline Minas Gerais & 3.809 & 282 & 6,89 & 873 & 632 & 41,99 & 12 & 14 & 53,85 & 42 & 50 & 54,35 \\
\hline Pará & 621 & 42 & 6,33 & 569 & 79 & 12,19 & 5 & 1 & 16,67 & 0 & 0 & SA \\
\hline Paraíba & 443 & 15 & 3,28 & 75 & 125 & 62,50 & 3 & 1 & 25,00 & 20 & 8 & 28,57 \\
\hline Paraná & 2.403 & 226 & 8,60 & 1.459 & 615 & 29,65 & 70 & 26 & 27,08 & 506 & 54 & 9,64 \\
\hline Pernambuco & 2.762 & 136 & 4,69 & 3.215 & 522 & 13,97 & 7 & 8 & 53,33 & 414 & 57 & 12,10 \\
\hline Piauí & 455 & 36 & 7,33 & 754 & 39 & 4,92 & 2 & 0 & 0,00 & 0 & 0 & SA \\
\hline Rio de Janeiro & 3.299 & 220 & 6,25 & 2.993 & 77 & 2,51 & 7 & 2 & 22,22 & 1.165 & 97 & 7,69 \\
\hline $\begin{array}{l}\text { Rio Grande do } \\
\text { Norte }\end{array}$ & 749 & 36 & 4,59 & 491 & 125 & 20,29 & 2 & 4 & 66,67 & 0 & 0 & SA \\
\hline $\begin{array}{l}\text { Rio Grande do } \\
\text { Sul }\end{array}$ & 1.618 & 274 & 14,48 & 1.367 & 805 & 37,06 & 42 & 13 & 23,64 & 453 & 99 & 17,93 \\
\hline Santa Catarina & 329 & 160 & 32,72 & 1.048 & 285 & 21,38 & 14 & 7 & 33,33 & 39 & 42 & 51,85 \\
\hline São Paulo & 7.930 & 1.004 & 11,24 & 4.336 & 4.756 & 52,31 & 98 & 56 & 36,36 & 3.963 & 453 & 10,26 \\
\hline Sergipe & 266 & 19 & 6,67 & 323 & 45 & 12,23 & 4 & 1 & 20,00 & 0 & 0 & SA \\
\hline Total & 31.531 & 2.902 & 8,43 & 24.549 & 9.848 & 28,63 & 310 & 147 & 32,17 & 7.005 & 930 & 11,72 \\
\hline
\end{tabular}

Fonte primária: Sistema Nacional de Transplantes, Ministério da Saúde (http://dtr2001.saude.gov.br/transplantes/).

Tx: transplantes realizados; L: pessoas em filas de espera; SA: sem atividade (não realizou transplantes do referido órgão e não possuía lista de espera).

acidental, e com o concomitante funcionamento dos órgãos que serão doados, após o obrigatório consentimento familiar. A morte encefálica deve ser devidamente diagnosticada por uma equipe médica, e o transplante, autorizado pelo SNT. Uma vez constatada por médicos a necessidade de transplante, o paciente candidato a receptor é colocado na fila. A fila para transplantes no SUS para cada órgão ou tecido é única, e o atendimento é por ordem de chegada, considerados critérios técnicos, geográficos, de compatibilidade e de urgência específicos para cada órgão, de acordo com a Portaria $n^{\circ} .91 /$ GM/MS 10, de 23 de janeiro de 2001. A fila é disciplinada pela Portaria $n^{\circ}$. 3.407/GM/MS 11, de 05 de agosto de 1998. Os critérios de urgência prevalecem, no caso do fígado, para o qual é adotado o critério baseado no Model for End-Stage Liver Disease (Meld)/ Pediatric End-Stage Liver Disease (Peld), regulamentado pela Portaria $n^{o} .1 .16012$ de 29 de maio de 2006 do Ministério da Saúde.

O SNT registra informações gerais sobre os transplantes de órgãos no Brasil, mas os transplantes realizados fora do SUS não são administrados pelo SNT. Cabe informar que, no sistema de saúde suplementar, os planos de saúde somente são obrigados a financiar transplantes de rim e de córnea, embora, eventualmente, paguem outros tipos de procedimentos, que são realizados nas suas redes referenciadas, que podem incluir hospitais públicos. Aspectos relacionados aos transplantes de órgãos realizados com finan- 
ciamento por instituições de saúde suplementar (os corriqueiramente chamados "planos" de saúde) são analisados em Bahia et al. 13. Os custos indiretos da não-realização de transplantes são elevados. Somente no caso dos rins, as terapias renais substitutivas, que podem, em grande medida, ser substituídas por transplantes, custaram aos cofres públicos, em 2005, a elevada cifra de $\mathrm{R} \$$ 1.159.679.058,23. Godoy et al. 14 estimam perdas de $11 \%$ na renda de portadores de doença renal crônica que, em grande parte, poderiam ser mitigadas por transplantes de rim. A essas perdas, devem ser adicionadas outras reconhecidas perdas de qualidade de vida dos pacientes em procedimentos de diálise, quando comparados aos pacientes transplantados.

Os órgãos não podem ser estocados por muito tempo (com exceção de ossos e medula) e somente podem ser utilizados uma vez. Um coração dura entre $4 \mathrm{~h}$ e $6 \mathrm{~h}$, um pulmão de $4 \mathrm{~h}$ a $6 \mathrm{~h}$, um pâncreas entre $12 \mathrm{~h}$ e $24 \mathrm{~h}$, um fígado entre $12 \mathrm{~h}$ e $24 \mathrm{~h}$ e um rim até $48 \mathrm{~h}$. Uma córnea pode durar até sete dias 15. Existe elevada "capacidade ociosa", pois há um desperdício considerável de órgãos. No Brasil, de cada oito potenciais doadores, apenas um é notificado e somente $20 \%$ deles são utilizados como doadores de múltiplos órgãos 15.

A despeito do reconhecimento da enorme magnitude das atividades públicas de transplantes no Brasil, o SNT convive com sérios problemas operacionais. Alguns desses problemas estão descritos em um recente relatório do Tribunal de Contas da União 16. No documento, são detalhados problemas de natureza gerencial, da qualidade e da atualização tecnológica dos procedimentos médicos e da garantia de prestação de tratamento tempestivo e equitativo ao público-alvo do SNT. Ribeiro \& Schramm 17 discutem aspectos morais importantes que deveriam ser reconhecidos na formulação de uma política brasileira de transplantes, diante das limitações de recursos no setor de saúde brasileiro, com a conseqüente necessidade de foco da atenção médica nas atividades relacionadas aos transplantes de órgãos em nosso país.

A importância da atuação do SNT é ampliada, pois as possibilidades de realização de transplantes com financiamento do sistema de saúde suplementar (planos de saúde) são limitadas. Tais limitações são de natureza legal e resultam da dinâmica de atuação dos referidos planos, conforme assinalam Bahia et al. 13. Um quadro preliminar comparativo do desempenho do SNT, com as atividades de transplantes em outros países, encontra-se na Tabela 1.

Na Tabela 2 vemos a dimensão e algumas disparidades entre os estados, nas filas para transplantes no SNT, no ano de 2006.
Cullis et al. 18 assinalam que as filas são um resultado dos descompassos entre a demanda e a oferta quando o sistema de preços não é o mecanismo determinante da produção e do consumo dos bens e produtos em saúde.

A demora no atendimento exerce impactos significativos sobre o bem-estar, as probabilidades de cura, a sobrevida dos enxertos e dos pacientes, a natureza e a extensão das seqüelas nos pacientes, nos familiares envolvidos, e na sociedade. Pior situação ocorre quando, além de elevados, os prazos são imprevisíveis. Com tal agravante, as incertezas decorrentes da imprevisibilidade impedem o planejamento das vidas dos pacientes e dos seus familiares, da atuação do sistema de saúde e do funcionamento do sistema produtivo onde eles por ventura trabalhem. Tempo de espera elevado implica, além dos custos e sofrimentos dos pacientes na fila, o aproveitamento de órgãos de qualidade inferior, e a conseqüente redução do tempo de duração dos enxertos. Daí advêm o aumento na necessidade de retransplantes, a provável elevação das taxas de mortalidade pós-transplantes e a redução da sobrevida atuarial da população transplantada ${ }^{19}$. Outro efeito deletério da espera é a crescente elevação do número de transplantes intervivos, que pode ter impacto significativo sobre a vida dos doadores, incluindo a esperada elevação dos custos dos planos de saúde privados para os mesmos. Embora bastante seguros e com melhores expectativas de resultados16, existe alguma evidência de que os transplantes intervivos (ao menos para o fígado) também são mais caros do que os realizados com doadores cadáveres 20 .

\section{Metodologia}

O número de transplantes realizados por ano, em cada Unidade da Federação, representa o equilíbrio possível e efetivo entre a taxa média de serviço (a oferta) e a taxa média de chegada de pacientes (a demanda) candidatos a transplantes no SNT. Em condições de total flexibilidade do "mercado" o número de transplantes realizados não poderia ser adotado como representativo da taxa de serviço, pois estaria configurado o conhecido "problema da identificação". Esse problema surge quando, na observação de um ponto de equilíbrio entre oferta e demanda em um mercado, não se pode dizer, a priori, se é possível resgatar a curva de oferta e a curva de demanda. Entretanto, no SNT, a taxa média de serviço é claramente restrita e menor do que a taxa de chegada, pois as filas existem, de modo que o sistema se equilibra sobre a própria taxa média de serviço (a oferta). 
O SNT disponibiliza em seu sítio na Internet a taxa média de serviço e o número de pessoas na fila para vários órgãos transplantados nos diversos estados e no Distrito Federal. Os dados disponíveis somente permitem associar as quantidades de transplantes realizados no ano de 2003 com as filas observadas em abril de 2004. As quantidades de transplantes realizados no ano de 2004 foram associadas às filas observadas em janeiro de 2005. Para o ano de 2006, os dados relacionados às filas e aos transplantes foram publicados na página do SNT na Internet.

$\mathrm{O}$ arcabouço analítico usado no presente estudo, baseado em elementos de queueing theory (para detalhes técnicos ver Gross \& Harris 21) é o mesmo utilizado em Marinho ${ }^{8}$, para o cálculo de filas. O modelo conhecido como Markovian/ Markovian/Single Model (M/M/1) é clássico e o mais simples disponível na literatura. Tal modelo recebe esse nome porque assume uma distribuição markoviana dos intervalos de chegada, que são dispostos de acordo com uma distribuição exponencial independente e identicamente distribuída (i.i.d.). A distribuição dos tempos de internação segue outro processo markoviano e uma outra distribuição exponencial também i.i.d. Como alternativa, existe a hipótese de que todos os pacientes tenham o mesmo tempo de tratamento, com a taxa de serviço $S$ seguindo uma distribuição degenerada, o que corresponderia ao modelo chamado de Markovian/Degenerate/ Single Model (M/D/1). Pode-se demonstrar que esse modelo e o modelo $\mathrm{M} / \mathrm{M} / 1$ representam os casos limites de um modelo mais geral, em que os tempos de tratamento seguem uma distribuição flexível do tipo Erlang com parâmetro k (modelo Markovian/Erlang/Single - M/ $\mathrm{E}_{\mathrm{k}} / 1$ ). Assim, o limite superior (no modelo $\mathrm{M} / \mathrm{M} / 1$ em que $\mathrm{k}=1$ ) e o limite inferior (no modelo $\mathrm{M} / \mathrm{D} / 1 \mathrm{em}$ que $\mathrm{k}$ = infinito) dos tempos de espera de todos os casos possíveis, ficam determinados. Ressalte-se que os resultados do tempo de espera nas filas obtidos no modelo M/M/1 são exatamente iguais ao dobro dos valores obtidos no modelo $\mathrm{M} / \mathrm{D} / 1$. O valor real do tempo de espera estaria entre os dois limites.

Os modelos baseiam-se, fundamentalmente, na interação entre duas variáveis: R é a taxa média de chegada de pacientes para transplantes que é a variável representativa da demanda por transplantes no SUS. S é a segunda variável, que representa a taxa média de serviço, ou seja, o número de transplantes por unidade de tempo, que é a variável representativa da oferta de serviços no sistema. Estimamos o tempo médio esperado na fila dos transplantes (exclui o tempo médio de internação): $\mathrm{Wq}=(\mathrm{R} / \mathrm{S}) /(\mathrm{S}-\mathrm{R})$.

\section{Resultados}

Na Tabela 3, e com o auxílio da Tabela 2 anterior, podemos descrever os resultados de nossas investigações. Os resultados para rim e córnea, que são os procedimentos mais freqüentes no SUS, podem também ser visualizados nas Figuras 1 e 2. Os resultados para os demais órgãos apresentam baixa freqüência nos estados estudados (dados não apresentados). Vale dizer que, no ano de 2006, apenas os estados de São Paulo (26 transplantes), Rio Grande do Sul (21 transplantes), Rio de Janeiro (4 transplantes) e Minas Gerais (2 transplantes) fizeram transplantes de pulmão. No que se refere ao pâncreas, o Estado de São Paulo fez 65 transplantes; o Estado de Minas Gerais fez 17 transplantes e o Rio Grande do Sul fez 2 transplantes. Para os transplantes simultâneos de rim/pâncreas, o Estado de São Paulo fez 65 transplantes, Minas Gerais fez 22 transplantes, o Rio Grande do Sul fez 20 transplantes e o Estado do Paraná fez 9 transplantes.

Constatamos que, em termos nacionais (com exceção do Estado do Acre, que foi retirado da amostra, por falta de registro de transplantes no período estudado), o tempo de espera para transplante de rim teria um limite inferior de 5,16 anos no ano de 2004, de 4,7 anos em 2005 e de 5,43 anos em 2006. Os limites superiores seriam, conforme citado na seção precedente, iguais ao dobro desses valores. Para efeitos de simplificação da apresentação dos resultados, adotamos a representação rim $(5,16 ; 4,7$ e 5,43) nos respectivos anos, indicando uma ligeira flutuação no tempo de espera. Calculamos, ainda, limites inferiores para a córnea $(1,51 ; 1,47$ e 1,25), que indicariam uma ligeira queda até o ano de 2006.

Para coração $(0,65 ; 0,64$ e 1,06), estimamos uma elevação do prazo de espera no período. Para o transplante de fígado (3,38; 3,44 e 3,77), o modelo estimou uma ligeira elevação do limite inferior do tempo de espera até 2006.

No caso do transplante de pulmão nos anos de 2004, 2005 e 2006, com respectivos tempos de espera $(1,14 ; 1,35$ e 1,03$)$, estimamos pouca variabilidade. Para o pâncreas $(1,77 ; 0,78$ e 0,68), ocorreu uma sensível redução do tempo de espera, enquanto para transplante de rim/pâncreas (0,9; 0,7 e 1,4), o modelo estimou uma ligeira redução inicial, seguida de uma elevação do limite inferior do prazo de espera.

No país, a quantidade total de transplantes realizados, nos órgãos aqui estudados, passou de 11.750 no ano de 2004, para 12.978 no ano de 2005, e para 14.100 em 2006, o que representa um aumento de $20 \%$ em relação ao ano inicial. A lista total de espera passou de 59.167 pessoas em 2004, para 61.119 pessoas em 2005 e 63.975 pes- 
Tabela 3

Tempo de espera (em anos) dos transplantes de órgãos de acordo com os modelos M/M/1 e M/D/1 por Unidade da Federação, Brasil, em $2004-2006$.

\begin{tabular}{|c|c|c|c|c|c|c|c|c|c|c|c|c|}
\hline \multirow{4}{*}{$\begin{array}{l}\text { Unidade da } \\
\text { Federação }\end{array}$} & \multicolumn{12}{|c|}{ Tempo de espera (anos) } \\
\hline & \multicolumn{6}{|c|}{$\operatorname{Rim}$} & \multicolumn{6}{|c|}{ Córnea } \\
\hline & \multicolumn{2}{|c|}{2004} & \multicolumn{2}{|c|}{2005} & \multicolumn{2}{|c|}{2006} & \multicolumn{2}{|c|}{2004} & \multicolumn{2}{|c|}{2005} & \multicolumn{2}{|c|}{2006} \\
\hline & Máximo & Mínimo & Máximo & Mínimo & Máximo & Mínimo & Máximo & Mínimo & Máximo & Mínimo & Máximo & Mínimo \\
\hline Alagoas & 20,47 & 10,24 & 26,04 & 13,02 & 28,20 & 14,10 & 21,20 & 10,60 & 22,60 & 11,30 & 15,00 & 7,50 \\
\hline Amazonas & 42,00 & 21,00 & 22,96 & 11,48 & 16,83 & 8,41 & 48,67 & 24,33 & 20,26 & 10,13 & 6,93 & 3,46 \\
\hline Bahia & 27,05 & 13,52 & 15,31 & 7,65 & 36,54 & 18,27 & 12,65 & 6,33 & 10,18 & 5,09 & 6,16 & 3,08 \\
\hline Ceará & 2,85 & 1,43 & 2,86 & 1,43 & 3,66 & 1,83 & 4,72 & 2,36 & 3,70 & 1,85 & 6,70 & 3,35 \\
\hline Distrito Federal & 16,47 & 8,23 & 40,00 & 20,00 & 15,50 & 7,75 & 6,97 & 3,49 & 6,11 & 3,06 & 4,38 & 2,19 \\
\hline Espírito Santo & 9,61 & 4,80 & 9,36 & 4,68 & 15,36 & 7,68 & 2,81 & 1,41 & 3,41 & 1,70 & 3,63 & 1,81 \\
\hline Goiás & 3,52 & 1,76 & 4,18 & 2,09 & 7,06 & 3,53 & 2,61 & 1,30 & 2,39 & 1,20 & 2,34 & 1,17 \\
\hline Maranhão & SA & SA & 0,00 & 0,00 & 13,03 & 6,52 & 238,00 & 119,00 & 67,75 & 33,87 & 6,21 & 3,10 \\
\hline Mato Grosso & 25,96 & 12,98 & 10,69 & 5,34 & 849,00 & 424,50 & 7,90 & 3,95 & 2,10 & 1,05 & 10,15 & 5,08 \\
\hline Mato Grosso do Sul & 4,47 & 2,23 & 17,82 & 8,91 & 5,57 & 2,79 & 0,73 & 0,36 & 1,87 & 0,93 & 1,64 & 0,82 \\
\hline Minas Gerais & 12,89 & 6,44 & 10,37 & 5,19 & 13,51 & 6,76 & 2,18 & 1,09 & 1,74 & 0,87 & 1,38 & 0,69 \\
\hline Pará & 25,86 & 12,93 & 12,12 & 6,06 & 14,81 & 7,40 & 5,66 & 2,83 & 7,68 & 3,84 & 7,22 & 3,61 \\
\hline Paraíba & 79,00 & 39,50 & 136,00 & 68,00 & 29,60 & 14,80 & 0,36 & 0,18 & 0,09 & 0,05 & 0,61 & 0,30 \\
\hline Paraná & 10,91 & 5,46 & 11,15 & 5,57 & 10,64 & 5,32 & 2,29 & 1,14 & 1,96 & 0,98 & 2,37 & 1,19 \\
\hline Pernambuco & 21,85 & 10,92 & 22,76 & 11,38 & 20,32 & 10,16 & 9,07 & 4,54 & 9,06 & 4,53 & 6,16 & 3,08 \\
\hline Piauí & 8,67 & 4,34 & 9,38 & 4,69 & 12,67 & 6,33 & 15,03 & 7,51 & 15,34 & 7,67 & 19,36 & 9,68 \\
\hline Rio de Janeiro & 10,38 & 5,19 & 11,68 & 5,84 & 15,00 & 7,50 & 9,49 & 4,74 & 17,24 & 8,62 & 38,88 & 19,44 \\
\hline Rio Grande do Norte & 28,85 & 14,42 & 20,28 & 10,14 & 20,83 & 10,42 & 5,02 & 2,51 & 3,38 & 1,69 & 3,94 & 1,97 \\
\hline Rio Grande do Sul & 4,22 & 2,11 & 6,86 & 3,43 & 5,91 & 2,95 & 1,92 & 0,96 & 3,48 & 1,74 & 1,70 & 0,85 \\
\hline Santa Catarina & 2,66 & 1,33 & 2,96 & 1,48 & 2,06 & 1,03 & 4,13 & 2,07 & 4,49 & 2,25 & 3,68 & 1,84 \\
\hline São Paulo & 10,09 & 5,04 & 6,94 & 3,47 & 7,90 & 3,95 & 1,64 & 0,82 & 1,54 & 0,77 & 0,91 & 0,46 \\
\hline Sergipe & 27,87 & 13,94 & 78,67 & 39,33 & 14,05 & 7,03 & 3,67 & 1,84 & 3,11 & 1,56 & 7,20 & 3,60 \\
\hline Total & 10,32 & 5,16 & 9,40 & 4,70 & 10,87 & 5,43 & 3,03 & 1,51 & 2,94 & 1,47 & 2,49 & 1,25 \\
\hline Unidade da & \multicolumn{12}{|c|}{ Tempo de espera (anos) } \\
\hline \multirow[t]{3}{*}{ Federação } & \multicolumn{6}{|c|}{ Coração } & \multicolumn{6}{|c|}{ Fígado } \\
\hline & \multicolumn{2}{|c|}{2004} & \multicolumn{2}{|c|}{2005} & \multicolumn{2}{|c|}{2006} & \multicolumn{2}{|c|}{2004} & \multicolumn{2}{|c|}{2005} & 20 & \\
\hline & Máximo & Mínimo & Máximo & Mínimo & Máximo & Mínimo & Máximo & Mínimo & Máximo & Mínimo & Máximo & Mínimo \\
\hline Alagoas & 4,83 & 2,41 & 0,81 & 0,40 & SA & SA & SA & SA & SA & SA & SA & SA \\
\hline Amazonas & SA & SA & SA & SA & SA & SA & SA & SA & SA & SA & SA & SA \\
\hline Bahia & SA & SA & SA & SA & SA & SA & 27,75 & 13,87 & 12,50 & 6,25 & 16,79 & 8,39 \\
\hline Ceará & 0,31 & 0,15 & 0,39 & 0,19 & 0,74 & 0,37 & 2,89 & 1,45 & 2,37 & 1,18 & 3,48 & 1,74 \\
\hline Distrito Federal & SA & SA & SA & SA & SA & SA & SA & SA & SA & SA & SA & SA \\
\hline Espírito Santo & 1,62 & 0,81 & SA & SA & SA & SA & SA & SA & SA & SA & 1,09 & 0,55 \\
\hline Goiás & 3,94 & 1,97 & 1,37 & 0,69 & 6,97 & 3,48 & SA & SA & SA & SA & SA & SA \\
\hline Maranhão & SA & SA & SA & SA & SA & SA & SA & SA & SA & SA & SA & SA \\
\hline Mato Grosso & SA & SA & SA & SA & SA & SA & SA & SA & SA & SA & SA & SA \\
\hline Mato Grosso do Sul & 15,94 & 7,97 & SA & SA & SA & SA & SA & SA & SA & SA & SA & SA \\
\hline Minas Gerais & 0,28 & 0,14 & 0,20 & 0,10 & 0,92 & 0,46 & 2,34 & 1,17 & 2,07 & 1,04 & 0,86 & 0,43 \\
\hline Pará & 0,00 & 0,00 & 2,41 & 1,21 & 5,85 & 2,93 & SA & SA & SA & SA & SA & SA \\
\hline Paraíba & SA & SA & SA & SA & 3,79 & 1,90 & SA & SA & 2,73 & 1,36 & 2,62 & 1,31 \\
\hline Paraná & 8,62 & 4,31 & 3,05 & 1,52 & 2,73 & 1,37 & 5,09 & 2,55 & 5,05 & 2,52 & 9,39 & 4,69 \\
\hline Pernambuco & 0,57 & 0,29 & 0,73 & 0,37 & 0,99 & 0,49 & 8,50 & 4,25 & 8,34 & 4,17 & 7,28 & 3,64 \\
\hline Piauí & 0,00 & 0,00 & 0,00 & 0,00 & SA & SA & SA & SA & SA & SA & SA & SA \\
\hline
\end{tabular}

(continua) 
Tabela 3 (continuação)

\begin{tabular}{|c|c|c|c|c|c|c|c|c|c|c|c|c|}
\hline \multirow{4}{*}{$\begin{array}{l}\text { Unidade da } \\
\text { Federação }\end{array}$} & \multicolumn{12}{|c|}{ Tempo de espera (anos) } \\
\hline & \multicolumn{6}{|c|}{ Coração } & \multicolumn{6}{|c|}{ Fígado } \\
\hline & \multicolumn{2}{|c|}{2004} & \multicolumn{2}{|c|}{2005} & \multicolumn{2}{|c|}{2006} & \multicolumn{2}{|c|}{2004} & \multicolumn{2}{|c|}{2005} & \multicolumn{2}{|c|}{2006} \\
\hline & Máximo & Mínimo & Máximo & Mínimo & Máximo & Mínimo & Máximo & Mínimo & Máximo & Mínimo & Máximo & Mínimo \\
\hline Rio de Janeiro & 1,58 & 0,79 & 3,44 & 1,72 & 3,94 & 1,97 & 11,10 & 5,55 & 13,11 & 6,55 & 12,02 & 6,01 \\
\hline Rio Grande do Norte & SA & SA & 2,73 & 1,37 & 0,68 & 0,34 & SA & SA & SA & SA & SA & SA \\
\hline Rio Grande do Sul & 2,50 & 1,25 & 12,32 & 6,16 & 3,31 & 1,65 & 2,34 & 1,17 & 4,86 & 2,43 & 4,59 & 2,29 \\
\hline Santa Catarina & 16,94 & 8,47 & 16,94 & 8,47 & 2,13 & 1,07 & 1,19 & 0,60 & 1,93 & 0,97 & 0,95 & 0,48 \\
\hline São Paulo & 0,75 & 0,37 & 0,79 & 0,39 & 1,77 & 0,88 & 8,57 & 4,29 & 7,48 & 3,74 & 8,75 & 4,38 \\
\hline Sergipe & SA & SA & SA & SA & 4,83 & 2,41 & SA & SA & SA & SA & SA & SA \\
\hline Total & 1,30 & 0,65 & 1,28 & 0,64 & 2,12 & 1,06 & 6,76 & 3,38 & 6,88 & 3,44 & 7,53 & 3,77 \\
\hline
\end{tabular}

Fonte primária: Sistema Nacional de Transplantes, Ministério da Saúde (http://dtr2001.saude.gov.br/transplantes/).

Máximo: tempo máximo de espera (modelo M/M/1); Mínimo: tempo mínimo de espera (modelo M/D/1); SA: sem atividade (não realizou transplantes do referido órgão e não possuía lista de espera).

soas em 2006, vale dizer, um aumento de aproximadamente $8 \%$ em relação ao ano de 2004.

Por outro lado, a Tabela 2 também explicita que a demanda total de transplantes, para cada tipo de órgão, ao final de cada ano, em cada estado, pode ser dividida em dois componentes básicos: os transplantes realizados (variável Tx na Tabela 2) e os transplantes ainda não realizados, isto é, as filas para transplantes (variável L na Tabela 2). Assim, um indicador natural do balanço entre o que foi realizado (Tx) e a demanda total em cada estado $(\mathrm{Tx}+\mathrm{L})$ é dado pela razão de atendimento $\mathrm{Tx} /(\mathrm{Tx}+\mathrm{L})$.

Vemos as disparidades das razão de atendimento para transplantes de rim, que vão de 0,12\% em Mato Grosso a 32,72\% em Santa Catarina (Tabela 2) no ano de 2006. A razão nacional média de atendimento é de $8,43 \%$. Também encontramos, na Tabela 3 e na Figura 1, tempos mínimos de espera que vão de elevados 424,5 anos no Mato Grosso a 1,03 anos em Santa Catarina. Note-se, na Tabela 2, que Mato Grosso fez apenas um transplante, e apresentava 848 pessoas na fila. No referido ano, a média nacional otimista de espera para rim é de 5,43 anos.

No caso dos transplantes de córnea verificamos, na Tabela 2, no ano de 2006, taxas de atendimento que vão de parcos 2,51\% no Rio de Janeiro, até $62,5 \%$ na Paraíba. A média nacional para esse indicador é de $28,63 \%$. Por outro lado, vemos, na Tabela 3 e na Figura 2, tempos de espera que vão de elevados 19,44 anos no Rio de Janeiro, até apenas 0,3 ano na Paraíba. A média nacional de espera é de 1,25 ano para transplantes de córnea.

\section{Discussão}

No conjunto das Unidades da Federação, fica evidente um predomínio do menor tempo de espera nas Regiões Sul e Sudeste, que também concentram a maior parte dos transplantes realizados e dos pacientes em filas. As exceções na Região Nordeste são os estados de Pernambuco e Ceará que se destacam positivamente, tanto por apresentarem tempos estimados de espera relativamente baixos, quanto por suas relativamente elevadas frequências de realização de transplantes (especialmente de rim, fígado e córnea). Na Região Sudeste, e no país, o destaque positivo é o Estado de São Paulo. Esse bom desempenho pode ser observado pelo exame do relativamente menor tempo de espera, em razão das elevadas freqüências de realização de todos os tipos de transplantes. O Estado de São Paulo realizou, no total do período estudado, 16.230 transplantes, em um total nacional de 38.828 transplantes, ou seja, aproximadamente $42 \%$ do total. São Paulo também se destaca pela redução do tempo de espera nos principais órgãos transplantados no país (tirante o de coração que aumentou de 0,4 ano em 2004 para 0,8 ano em 2006, e de rim/pâncreas, com prazo praticamente estagnado em torno de 1,8 ano). O sistema de transplantes em São Paulo está bem relatado em Pereira et al. 22. Observa-se, ainda, a melhoria de sua posição relativa no tempo de espera em geral no país.

Um estado destoante em termos negativos, na Região Sudeste, é o Rio de Janeiro, cuja situação problemática já foi relatada em Viera ${ }^{23}$. A posição do estado, avaliada pelo tempo de espera, no conjunto da federação, é ruim na maior parte 
Tempo de espera mínimo (modelo M/D/1) para transplantes de rim por Unidade da Federação, Brasil, no ano 2006.

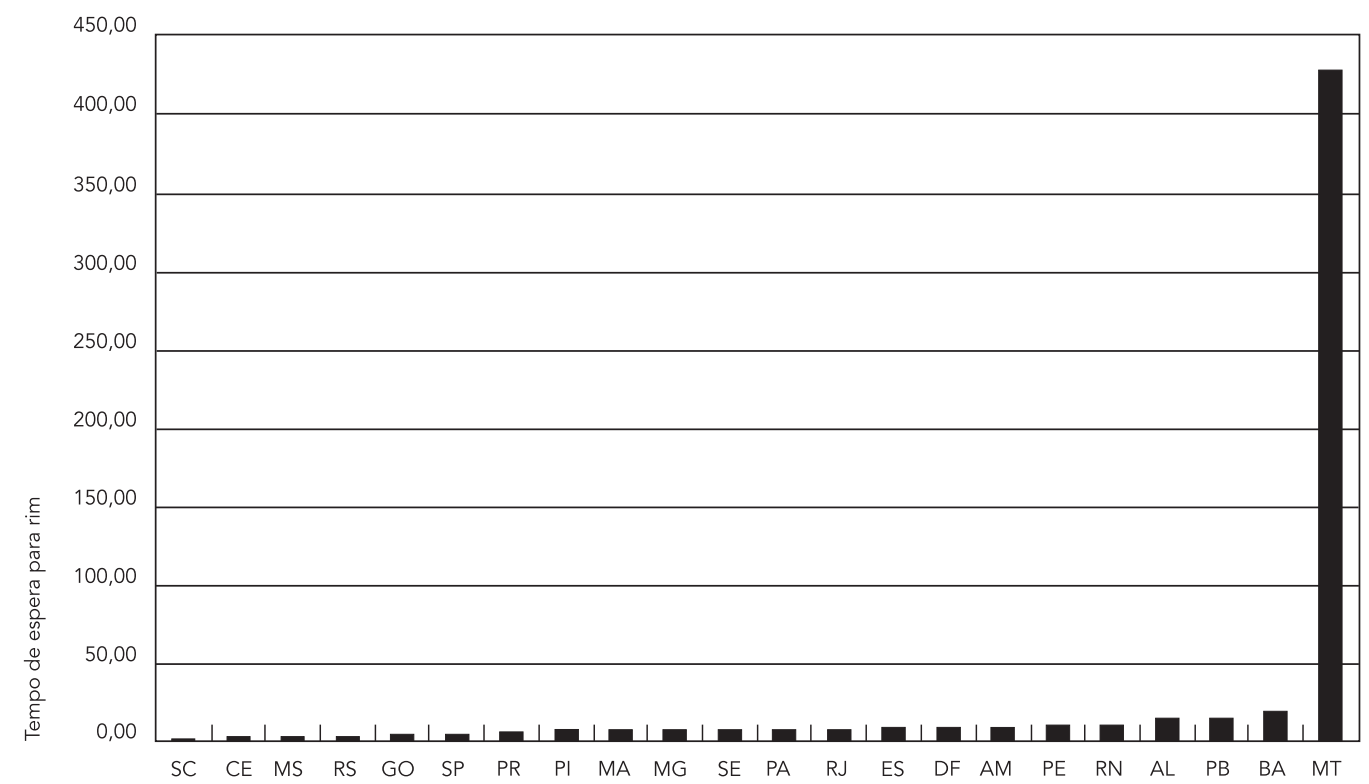

Unidades da Federação: AL: Alagoas; AM: Amazonas; BA: Bahia; CE: Ceará; DF: Distrito Federal; ES: Espírito Santo; GO: Goiás; MA: Maranhão; MT: Mato Grosso; MS: Mato Grosso do Sul; MG: Minas Gerais; PA: Pará; PB: Paraíba; PR: Paraná; PE: Pernambuco; PI: Piauí; RJ: Rio de Janeiro; RN: Rio Grande do Norte; RS: Rio Grande do Sul; SC: Santa Catarina; SP: São Paulo; SE: Sergipe.

dos órgãos analisados, salvo o de transplante de pulmão, que apresenta os menores prazos de espera do país. Mas ressalve-se que, nesse caso, apenas quatro estados (São Paulo, Rio Grande do Sul, Rio de Janeiro e Minas Gerais) realizaram transplantes (135 procedimentos no total) nos três anos analisados e que o Rio de Janeiro realizou apenas 14 desses transplantes. Além disso, o Estado do Rio de Janeiro piorou a sua posição relativa em praticamente todos os órgãos pesquisados (com exceção já citada de transplantes de pulmão).

O Rio de Janeiro teve o maior tempo de espera para transplante de córnea no ano de 2006 (19,4 anos, no modelo otimista), o que significa que, praticamente, essa atividade não é realizada na rede pública ligada ao SNT no referido estado.

Verificamos ainda, na Tabela 2, que o Estado de São Paulo realizou, no ano de 2006, um total de 4.756 transplantes de córnea, com 4.336 pessoas na fila, o que corresponde a um atendimento de 52,31\% da demanda total (a demanda total, como vimos, é a soma dos transplantes realizados com a quantidade de pessoas na fila de espera). O Estado do Rio de Janeiro, por sua vez, com 2.993 pessoas na fila, realizou apenas 77 transplantes de córnea, isto é, atendeu somente $2,51 \%$ da demanda total, obtendo o mais baixo índice do país. No Brasil como um todo, a taxa de atendimento para transplante de córnea é de $28,63 \%$.

Uma situação também desfavorável para o Estado do Rio de Janeiro ocorreu no transplante de rim. O Estado de São Paulo, com 7.930 pessoas na fila, realizou 1.004 transplantes, obtendo uma taxa de atendimento de 11,24\%. O Rio de Janeiro, com 3.299 pessoas na fila, fez 220 transplantes, o que corresponde a uma taxa de atendimento de $6,25 \%$. A taxa de atendimento para rim, no Brasil, é de $8,43 \%$.

O Rio de Janeiro tem, ainda, a segunda pior taxa de atendimento nos transplantes de fígado $(7,69 \%)$, à frente apenas do Estado da Bahia (5,65\%), para um indicador nacional de $11,72 \%$. Tais resultados negativos para o Estado do Rio de Janeiro não se coadunam com a capacidade econômica nem com a reconhecida pujança de recursos sanitários desse estado. 
Figura 2

Tempo de espera mínimo (modelo M/D/1) para transplantes de córnea por Unidade da Federação, Brasil, no ano de 2006.

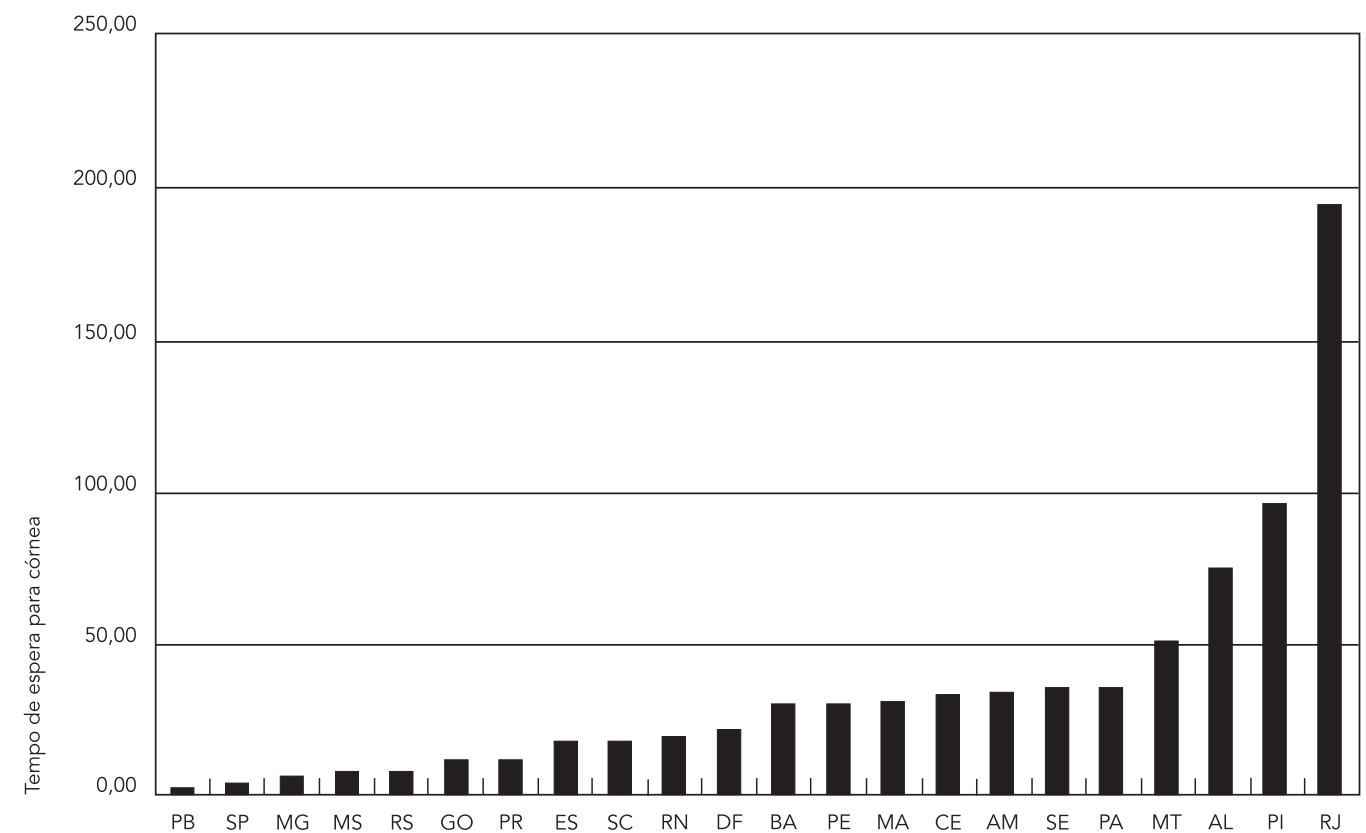

Unidades da Federação: AL: Alagoas; AM: Amazonas; BA: Bahia; CE: Ceará; DF: Distrito Federal; ES: Espírito Santo; GO: Goiás; MA: Maranhão; MT: Mato Grosso; MS: Mato Grosso do Sul; MG: Minas Gerais; PA: Pará; PB: Paraíba; PR: Paraná; PE: Pernambuco; PI: Piauí; RJ: Rio de Janeiro; RN: Rio Grande do Norte; RS: Rio Grande do Sul; SC: Santa Catarina; SP: São Paulo; SE: Sergipe.

Reconhecemos a necessidade de detalhar, estender e aprofundar a análise realizada no presente texto. Para isso, seria necessário, entretanto, incorporar dados e informações que não estão disponíveis no momento. Contudo, acreditamos que o presente estudo pode ser útil na análise do quadro atual e na formulação de políticas relacionadas aos transplantes de órgãos no Brasil. 


\section{Resumo}

Avaliamos alguns aspectos dos transplantes de órgãos nas Unidades da Federação brasileira, nos anos de 2004, 2005 e 2006. Estimamos, com base em um modelo de teoria das filas, os tempos de espera para transplantes de coração, córnea, fígado, pulmão, rim, pâncreas, e transplante simultâneo de rim e pâncreas. Os resultados indicam redução na espera por alguns órgãos (córnea, e pâncreas); elevação em outros (fígado, coração, rim/pâncreas); e ligeiras flutuações, sem tendência muito definida, nos transplantes de rim e nos transplantes de pulmão ao longo do período estudado. Os estados das regiões Sul e Sudeste (com a exceção do Rio de Janeiro) e Centro-oeste têm os menores tempos de espera e as maiores taxas de atendimento do país.

Transplante de Órgãos; Disparidades em Assistência à Saúde; Política de Saúde

\section{Colaboradores}

A. Marinho participou da elaboração do texto, escolha dos modelos de análise e avaliação dos resultados. S. S. Cardoso contribuiu na coleta de dados, elaboração dos modelos e cálculos e revisão geral do texto. V.V. Almeida colaborou na elaboração dos cálculos, pesquisa bibliográfica e revisão geral do texto.

\section{Agradecimentos}

Os autores agradecem os comentários de dois pareceristas anônimos. Os eventuais erros remanescentes são de nossa inteira responsabilidade.

\section{Referências}

1. Morris PJ, Monaco AP. Geographic disparities in access to organ transplantation. Transplantation 2003; 76:1383.

2. Chapman J, Russ G. Geographic variance in access to renal transplantation in Australia. Transplantation 2003; 76:1403-6.

3. Miranda B, Cañón J, Cuende N, Garrido G, Naya MT, Fernández-Zincke E. Disparities in access to liver transplantation in Spain. Transplantation 2003; 76:1398-403.

4. Ellison MD, Edwards LB, Edwards EB, Barker CF. Geographic differences in access to transplantation in the United States. Transplantation 2003; 76:1389-94.

5. Meltzer D. Waiting for organ transplantation. Transplant Proc 2003; 35:969-70.

6. Roudot-Thoraval F, Romano P, Spaak F, Houssin D, Durand-Zaleski I, Ellison MD, et al. Geographic disparities in access to organ transplantation in France. Transplantation 2003; 76:1385-8

7. Rudge CJ, Fuggle SV, Burbidge KM. Geographic disparities in access to organ transplantation in the United Kingdom. Transplantation 2003; 76:1395-8.
8. Marinho A. Um estudo sobre as filas para transplantes no Sistema Único de Saúde Brasileiro. Cad Saúde Pública 2006; 22:2229-39.

9. Joint Commission on Accreditation of Healthcare Organizations. Health care at crossroads: strategies for narrowing the organ donation gap and protecting patients. Washington DC: Joint Commission on Accreditation of Healthcare Organizations; 2004.

10. Brasil. Portaria no ${ }^{\circ}$ 91/GM/MS. Diário Oficial da União 2001; 25 jan.

11. Brasil. Portaria $n^{\circ} .3 .407 / G M / M S$. Diário Oficial da União 1998; 6 ago.

12. Brasil. Portaria nº. 1.160. Diário Oficial da União 2006; 31 mai.

13. Bahia L, Simmer E, Oliveira DC. Coberturas de planos privados de saúde e doenças crônicas: notas sobre utilização de procedimentos de alto custo. Ciênc Saúde Coletiva 2004; 9:921-9.

14. Godoy MR, Balbinotto Neto G, Ribeiro EP. Earnings and chronic renal disease. Transplant Proc 2007; 39:378-80. 
15. Associação Brasileira de Transplante de Órgãos, Ministério da Saúde, Conselho Federal de Medicina. Entenda a doação de órgãos: decida-se pela vida. http://www.abto.org.br/abtov02/portugues/ populacao/doacaoOrgaosTecidos/pdf/entenda doacao.pdf (acessado em 19/Jun/2009).

16. Tribunal de Contas da União. Relatório de avaliação de programa: Programa Doação, Captação e Transplante de Órgãos e Tecidos. Brasília: Tribunal de Contas da União, Secretaria de Fiscalização e Avaliação de Programas de Governo; 2006.

17. Ribeiro CDM, Schramm FR. Atenção médica, transplante de órgão e tecidos e políticas de focalização. Cad Saúde Pública 2006; 22:1945-53.

18. Cullis JG, Jones PR, Propper C. Waiting lists and medical care treatment: analysis and policies. In: Culyer AJ, Newhouse JP, editors. Handbook of health economics. Amsterdam: Elsevier North Holland; 2000. p. 1201-49.

19. Garcia VD, Abbud Filho M, Neumann J, Pestana JOM. Transplante de órgãos e tecidos. 2a Ed. São Paulo: Editora Segmento Farma; 2006.
20. Coelho JCU, Trubian PS, Freitas ACT, Parolin MB, Schulz GJ, Martins EL. Comparação entre o custo do transplante hepático cadavérico e o intervivos. Rev Assoc Med Bras 2005; 51:158-63.

21. Gross D, Harris CM. Fundamentals of queueing theory. 3rd Ed. New York: John Wiley \& Sons; 1998.

22. Pereira LA, Coria SA, Monteiro F, Scandiuzzi MC. Sistema estadual de transplantes em São Paulo: histórico, resultados e perspectivas. In: Bittar OJNV, Cecílio MAM, organizadores. Planejamento de saúde: conhecimento e ações, 2006. São Paulo: Secretaria de Estado de Saúde de São Paulo; 2006. p. 79-116.

23. Vieira M. A agonia dos transplantes no Rio: HU na imprensa. http://www.hucff.ufrj.br/na imprensa/2002/transplante.html (acessado em 26/Ago/2004).

24. NHS Blood and Transplant. Waiting time to transplant. http://www.uktransplant.org.uk/ukt/ about_transplants/waiting_time_to_transplant/ waiting_time_to_transplant.jsp (acessado em 03/ Jun/2009).

Recebido em 12/Jun/2009

Versão final reapresentada em 18/Dez/2009

Aprovado em 02/Fev/2010 